University of Nebraska - Lincoln

DigitalCommons@University of Nebraska - Lincoln

Anthony F. Starace Publications

Research Papers in Physics and Astronomy

March 1999

\title{
Magnetic-Field-Induced Intensity Revivals in Harmonic Generation
}

Dejan B. Miloševic

University of Nebraska - Lincoln

Anthony F. Starace

University of Nebraska-Lincoln, astarace1@unl.edu

Follow this and additional works at: https://digitalcommons.unl.edu/physicsstarace

Part of the Physics Commons

Miloševic, Dejan B. and Starace, Anthony F., "Magnetic-Field-Induced Intensity Revivals in Harmonic Generation" (1999). Anthony F. Starace Publications. 69.

https://digitalcommons.unl.edu/physicsstarace/69

This Article is brought to you for free and open access by the Research Papers in Physics and Astronomy at DigitalCommons@University of Nebraska - Lincoln. It has been accepted for inclusion in Anthony F. Starace Publications by an authorized administrator of DigitalCommons@University of Nebraska - Lincoln. 


\title{
Magnetic-Field-Induced Intensity Revivals in Harmonic Generation
}

\author{
Dejan B. Milošević* and Anthony F. Starace \\ Department of Physics and Astronomy, The University of Nebraska, Lincoln, Nebraska 68588-0111
}

(Received 10 December 1998)

\begin{abstract}
We demonstrate control of high harmonic generation by a linearly polarized laser field using a uniform static magnetic field parallel to the laser polarization. We predict that particular values of the magnetic field can increase harmonic intensities by orders of magnitude. Classical orbit calculations show that these magnetic-field-induced intensity revivals occur when the return time for laser-driven motion of the electron back to the origin is a multiple of the cyclotron period for motion perpendicular to the laser polarization direction. [S0031-9007(99)08759-1]
\end{abstract}

PACS numbers: $32.80 . \mathrm{Qk}, 32.80 . \mathrm{Wr}, 42.50 . \mathrm{Hz}, 42.65 . \mathrm{Ky}$

High harmonic generation (HHG) is a rapidly developing field of laser atomic physics, due mainly to the potential applications of the emitted high-brilliance, coherent, high-frequency radiation [1,2]. The key features of this process, its extended plateau, which consists of many harmonics with comparable intensities, and its sharp high-frequency cutoff, are explained using a "three-step" physical model [3,4]. According to this model, the "first step" is ionization, the "second step" is laser-driven propagation of the free electron, and the "third step" is the recollision of the electron with the atomic core, during which the electron can recombine and emit a harmonic photon. This model predicts the maximum energy of the harmonics to be $N_{\max } \hbar \omega=I_{p}+3.17 U_{p}$, where $I_{p}$ is the atomic ionization potential, $U_{p}=e^{2} E_{L}^{2} /\left(4 m_{e} \omega^{2}\right)$ is the ponderomotive energy, $-e$ and $m_{e}$ are the electron charge and mass, and $E_{L}$ and $\omega$ are the laser amplitude and frequency. The cutoff value, $N_{\max }$, was also predicted by quantum-mechanical theories [5]. Besides the position of the cutoff, another important characteristic of the HHG process is its efficiency, which is determined by the intensities of the harmonics on the plateau.

Demonstrated methods for controlling HHG include changing the polarization of the laser field [6], adding a second frequency component to the laser field [7], or adding a static electric [8] or magnetic field $[9,10]$. With regard to control of HHG with a static magnetic field, Zuo et al. [9] noted that the intensity of emitted harmonics is diminished by the spreading of the electron wave packet prior to its recollision with the atomic core. They proposed the use of a strong magnetic field to confine this spreading and demonstrated, for a two-color calculation, modest enhancement of HHG intensities for only one value of the magnetic induction, $B=0.2 B_{0}=47000 \mathrm{~T}$, where $B_{0}=\hbar /\left(e a_{0}^{2}\right)=2.3505 \times 10^{5} \mathrm{~T}$. This value of $B$ is much larger than the maximum presently achievable laboratory magnetic field (see below). Connerade and Keitel [10] also considered theoretically HHG in a strong magnetic field for the case of an intense pump laser. They focused on the possibility of generating even harmonics owing to relativistic motion of the laser-driven electron in the static magnetic field.

0031-9007/99/82(13)/2653(4)\$15.00
We consider here theoretically the control of HHG with a strong static magnetic field directed along the $z$ axis whose magnitude is comparable to those presently achievable. We assume the use of a laser linearly polarized along the $z$ axis whose intensity is moderate so that relativistic effects may be ignored. For selected values of $B$, we show that particular harmonics increase in intensity by $2-3$ orders of magnitude. For selected harmonics, we demonstrate that the intensity is a strong function of the value $B$ with periodic revivals of intensity. We have also carried out classical calculations of the active electron's motion in the driving laser field in order to interpret the quantum-mechanical results obtained.

We restrict ourselves to values of $B$ that are large, but nevertheless of an order of magnitude currently achievable experimentally. Kudasov et al. [11] have reported experiments which obtain "reproducible uniform magnetic fields with $B$ up to $1000 \mathrm{~T}$ in large useful volumes." The "useful volume" in Ref. [11] consists of a cylinder approximately $10 \mathrm{~mm}$ in diameter and $100 \mathrm{~mm}$ long. The duration of the magnetic pulses is a few $\mu$ s, which is much larger than typical laser field pulse durations, so that one can consider the magnetic field as constant. The present calculations are carried out for a $5 \times 10^{10} \mathrm{~W} / \mathrm{cm}^{2}$ $\mathrm{CO}_{2}$ laser, whose frequency is $\hbar \omega=0.1165 \mathrm{eV}$. For simplicity we present our results for the $\mathrm{H}^{-}$negative ion, for which there exist good variational representations for the initial state wave functions [12]. Phenomena similar to those reported here for $\mathrm{H}^{-}$should occur also for neutral atom targets [13]. Finally, we ignore the effect of the magnetic field on the $\mathrm{H}^{-}$ground state wave function. Even for $B=9000 \mathrm{~T}$, the ground state wave function is essentially unchanged for hyperspherical radii $R=\sqrt{r_{1}^{2}+r_{2}^{2}} \leq 6$, within which the peak amplitude is located [14]. Only the tail of the wave function for $R>6$ is changed slightly owing to the magnetic field compression [14].

We obtain harmonic spectra by computing the quantum-mechanical dipole moment $\mathbf{D}_{N}$, which is defined by the Fourier transform of the time-dependent dipole 
matrix element $\mathbf{d}(t)$,

$\mathbf{D}_{N}=\int_{0}^{T} \frac{d t}{T} \mathbf{d}(t) \exp (i N \omega t), \quad \mathbf{d}(t)=\langle\Phi(t)|e \mathbf{r}| \Phi(t)\rangle$,

where $T=2 \pi / \omega$ and $|\Phi(t)\rangle$ is the solution of the Schrödinger equation for the system (atom plus laser field plus magnetic field). Using the strong-field approximation and neglecting the continuum-continuum coupling, $\mathbf{d}(t)$ can be written as [5]

$$
\mathbf{d}(t)=\int_{-\infty}^{t} d t^{\prime}\left\langle u(t)\left|e \mathbf{r} G_{L}\left(t, t^{\prime}\right) e \mathbf{E}_{L}\left(t^{\prime}\right) \cdot \mathbf{r}\right| u\left(t^{\prime}\right)\right\rangle+\text { c.c. }
$$

where $e \mathbf{E}_{L}\left(t^{\prime}\right) \cdot \mathbf{r}$ is the interaction of the electron with the laser field in the length gauge, $|u(t)\rangle=\left|u_{0}\right\rangle \exp \left(i I_{p} t / \hbar\right)$ is the atomic ground state, and $G_{L}\left(t, t^{\prime}\right)$ is a Volkov-type Green's function for the electron in the presence of the laser field and the magnetic field,

$$
G_{L}\left(\mathbf{r}, t ; \mathbf{r}^{\prime}, t^{\prime}\right)=-\frac{i}{\hbar} \theta\left(t-t^{\prime}\right) \int_{-\infty}^{\infty} d q \sum_{n=0}^{\infty} \sum_{m=-\infty}^{\infty} \Psi_{n m q}(\rho, \phi, z, t) \Psi_{n m q}^{*}\left(\rho^{\prime}, \phi^{\prime}, z^{\prime}, t^{\prime}\right)
$$

and $\Psi_{n m q}(\rho, \phi, z, t)$ is a solution of the Schrödinger equation for the electron in the presence of both the laser field and the static magnetic field in cylindrical coordinates, $\mathbf{r} \equiv(\rho, \phi, z)$. Although we ignore the effect of the magnetic field on the initial state of the electron, it is included in $G_{L}\left(t, t^{\prime}\right)$ and hence it has an influence on the electron's intermediate states. In this paper we consider the case of parallel fields $\left(\mathbf{E}_{L} \| \mathbf{B}\right)$ along the $z$ axis, for which Eqs. (2) and (3) can be simplified [13]. The summations over the quantum numbers $n$ and $m$ can be performed analytically; the integral over the $z$ component of the intermediate electron momentum is computed using the saddle-point method, as in Ref. [5].

Figure 1 shows the harmonic intensity (i.e., the efficiency, defined by $\left|D_{N}\right|^{2}$ ) for a $5 \times 10^{10} \mathrm{~W} / \mathrm{cm}^{2} \mathrm{CO}_{2}$ laser as a function of the harmonic order $N$ for three different values of the magnetic induction: $B=0 \mathrm{~T}$ (squares), $1000 \mathrm{~T}$ (triangles), and $2000 \mathrm{~T}$ (circles). For the $\mathrm{H}^{-}$ion, $I_{p}=0.754 \mathrm{eV}$ for $B=0$, so that $N_{\max } \approx 21$, which agrees with the results presented in Fig. 1 in the absence of the magnetic field. In the presence of the magnetic field, one should take into account the magneticfield-induced threshold shift, $\frac{1}{2} \hbar \omega_{B}$, where $\omega_{B}=e B / m_{e}$,

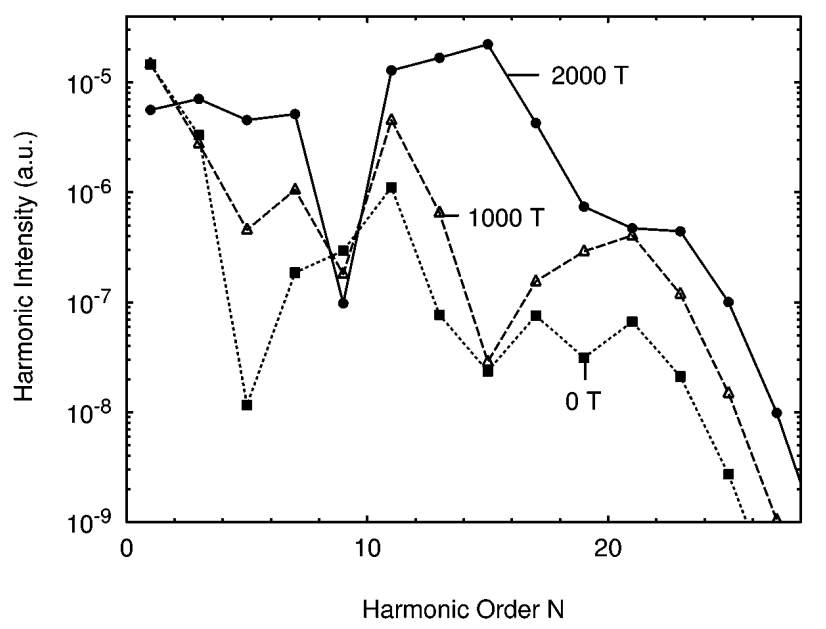

FIG. 1. Harmonic intensities as functions of the harmonic order $N$ for the $\mathrm{H}^{-}$ion in a $\mathrm{CO}_{2}$ laser with intensity $I_{L}=$ $5 \times 10^{10} \mathrm{~W} / \mathrm{cm}^{2}$. The magnetic field induction is $B=0 \mathrm{~T}$ (squares), $1000 \mathrm{~T}$ (triangles), and $2000 \mathrm{~T}$ (circles). which leads to a slight increase in $N_{\max }$ (by about 1 for $B=2000 \mathrm{~T}$ ). Only the odd harmonics are generated because the magnetic field preserves axial symmetry. For $B=2000 \mathrm{~T}$ there is a pronounced maximum of the harmonic intensity at about $N=15$.

Figure 2 shows the odd harmonic intensities for $11 \leq$ $N \leq 19$ as functions of $B$. Figure 2(a) shows that there are pronounced maxima for $B \approx 2000,4000,6000$, and $8000 \mathrm{~T}$. Clearly, there are optimum values of $B$ for which the intensity of a fixed harmonic has maxima. Figure 2(b) shows on an expanded scale the dependence of the intensity of the 15th harmonic on $B$ for $50 \leq B \leq 500 \mathrm{~T}$. Once again, one observes distinct peaks of intensity for particular values of $B$ although all of the peaks in Fig. 2(b) are of lower intensity than the one shown for the 15th harmonic in Fig. 2(a) near $B=2000 \mathrm{~T}$.

An interpretation of our quantum mechanical results in Figs. 1 and 2 may be obtained from classical considerations using the three-step model $[3,4]$. According to this classical model, the electron is born at time $t_{0}$ at the origin $\left[\mathbf{r}\left(t_{0}\right)=\mathbf{0}\right]$ with zero initial momentum. It then moves under the influence of both the laser and static magnetic fields. Solving Newton's equation for the electron, $m_{e} \ddot{\mathbf{r}}=-e\left[\mathbf{E}_{L}(t)+\dot{\mathbf{r}} \times \mathbf{B}\right]$, one obtains the electron kinetic energy $E_{k}$ at the time $t_{1}$ when the electron returns to the origin. The maximum of this energy and, therefore, the cutoff energy, $N_{\max } \hbar \omega=I_{p}+E_{k, \max }$, is determined by the two following conditions: $\mathbf{r}\left(t_{1}\right)=\mathbf{0}$ (i.e., the electron, after the return time $\tau=t_{1}-t_{0}$, comes back to the atomic core) and $\partial E_{k} / \partial t_{0}=0$ (i.e., the optimum time $t_{0}$ at which the electron is born for which $E_{k}$ has a maximum). For a linearly polarized laser field $\mathbf{E}_{L}(t)=E_{L} \sin \omega t \hat{\mathbf{z}}$ and a parallel magnetic field $\mathbf{B}=B \hat{\mathbf{z}}$, Newton's equation separates and, for zero initial momentum, we have $m_{e} \dot{x}=e B y, m_{e} \dot{y}=-e B x$, and $m_{e} \dot{z}=e\left[A_{L}(t)-A_{L}\left(t_{0}\right)\right]$, so that $E_{k}=m_{e} \dot{\mathbf{r}}^{2}(t) / 2=$ $e^{2}\left\{\left[\mathbf{A}_{L}(t)-\mathbf{A}_{L}\left(t_{0}\right)\right]^{2}+B^{2}\left(x^{2}+y^{2}\right)\right\} /\left(2 m_{e}\right)$. The electron rotates in the plane perpendicular to the magnetic field with the cyclotron period $\tau_{B}=2 \pi / \omega_{B}$, while its parallel motion is determined by the laser field only. If $t_{1}=t_{0}+\tau_{B}, t_{0}+2 \tau_{B}, \ldots$, at the time the electron returns to the origin, $z\left(t_{1}\right)=0$, then the return time $\tau=t_{1}-t_{0}$ is a multiple of the cyclotron period $\tau_{B}$. 

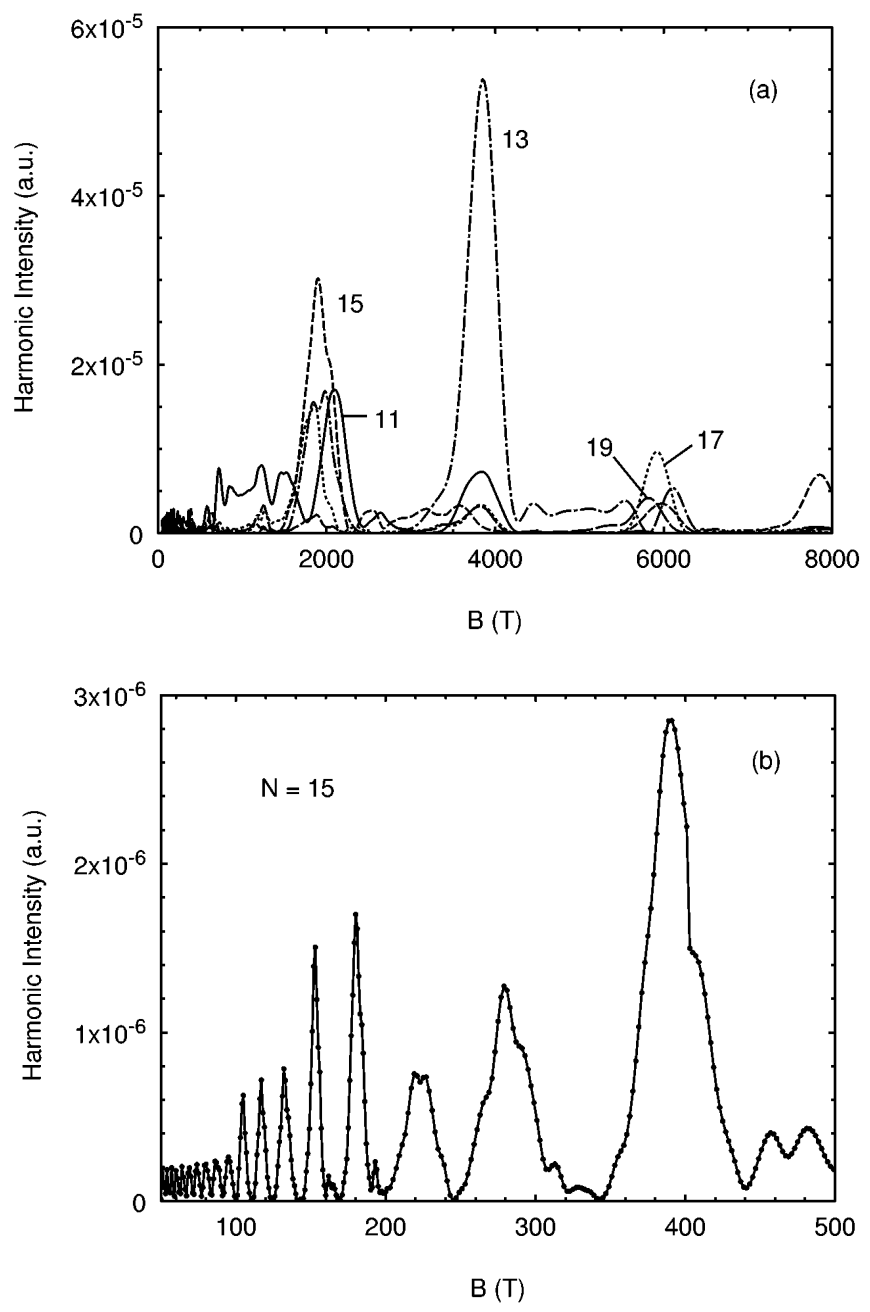

FIG. 2. Harmonic intensities vs $B$ for fixed harmonic order: $N=11$ (solid line), 13 (dot-dashed line), 15 (dashed line), 17 (dotted line), and 19 (long-dashed line). The laser field and the $\mathrm{H}^{-}$ion parameters are the same as in Fig. 1. (a) $0 \leq B \leq 8000 \mathrm{~T}$; (b) $50 \leq B \leq 500 \mathrm{~T}$.

Quantum mechanically, this classical prediction implies that the electron's wave packet for motion along the $z$ axis under the influence of the laser field is coincident with the magnetic-field-induced oscillation of the electronic wave packet perpendicular to the $z$ axis. The solution of the equation $\mathbf{r}\left(t_{1}\right)=\mathbf{0}$ leads to $E_{k}=8 U_{p} a^{2} b^{2} /\left(a^{2}+b^{2}\right)$, where $a(\sigma)=\sin \sigma, \quad b(\sigma)=\cos \sigma-\sin \sigma / \sigma$, and $\sigma=\omega \tau / 2$, so that $E_{k, \max }=3.17 U_{p}$. Useful information about the HHG process can be obtained by presenting $E_{k}$ as a function of the return time $\tau$ [5].

In Fig. 3 we present classical calculations of the kinetic energy of the electron at the origin, $E_{k}$, as a function of the return time, $\tau$. However, we do so in an indirect way. Instead of $E_{k}$, we plot the harmonic order $N \equiv$ $\left(E_{k}+I_{p}\right) / \hbar \omega$. Also, instead of $\tau$, we plot the value of $B$ corresponding to a cyclotron period, $\tau_{B}$, equal to $\tau$, i.e., $\tau=\tau_{B}=2 \pi / \omega_{B}=2 \pi m_{e} /(e B)$ or $B=2 \pi m_{e} /(e \tau)$. The shortest return times thus correspond to the largest magnetic fields $B$. The intersections of the horizontal lines shown for the harmonics $11 \leq N \leq 19$ with the classical curve $N(\tau)$ indicate particular return times for each harmonic. Thus, for $N=15$, the shortest return time corresponds to one cyclotron period $\tau_{B}$ for $B=2200 \mathrm{~T}$; the next return time (corresponding to two laser driving periods) corresponds to one cyclotron period $\tau_{B}$ for $B \approx 1200 \mathrm{~T}$; longer and longer return times (corresponding to increasingly many electron excursions up and down the $z$ axis under the influence of the laser field) correspond to one cyclotron period $\tau_{B}$ for smaller and smaller $B$ fields. Interestingly near $B \approx 850 \mathrm{~T}$, the $N>13$ harmonics do not have a classical orbit solution which returns to the origin, while the $N \leq 13$ harmonics do. We conclude that the various classical orbits the electron makes under the influence of the laser field correspond to one cyclotron period $\tau_{B}$ for a wide range of values of $B$, with the shortest return times corresponding to the largest $B$ values and the longest return times corresponding to increasingly small, densely spaced values of $B$.

In Fig. 4 we combine our quantum mechanical calculations with our classical orbit calculations in order to interpret our theoretical predictions of magnetic-field-induced intensity revivals in harmonic generation. This figure is similar to Fig. 2(a), but it is not the same. Rather, for each of the odd harmonics $15 \leq N \leq 19$, we have carried out classical calculations for the first return time, $\tau_{1}(N)$, while for $N=11,13$ we have calculated the second return time $\tau_{2}(N)$. In Fig. $3 \tau_{1}(N)\left[\tau_{2}(N)\right]$ would be equal to the first (second) intersection (from the right) of the appropriate horizontal line with the curve. We now plot our quantum mechanical results for the intensity of the harmonics $11 \leq$ $N \leq 19$ vs $B$ in terms of $\tau_{i}(N) / \tau_{B} \equiv\left[e \tau_{i}(N) /\left(2 \pi m_{e}\right)\right] B$, for $i=1,2$. Classically, the motion along the $z$ axis is independent of $B$ and hence so is $\tau_{i}(N)$; the linear dependence of $\tau_{i}(N) / \tau_{B}$ on $B$ stems from the division of $\tau_{i}(N)$

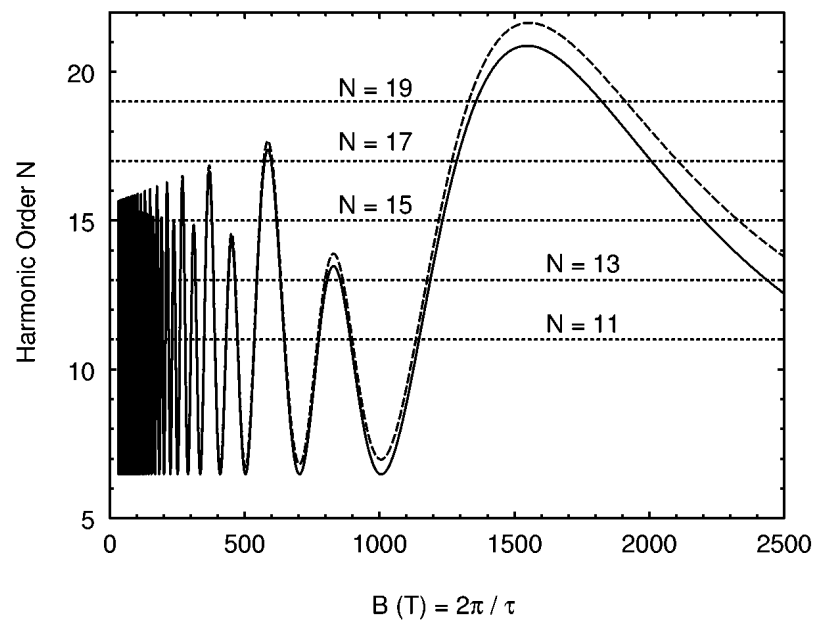

FIG. 3. Harmonic order $N$ vs the magnetic induction $B$ for which one cyclotron period, $\tau_{B}$, equals the classical return time $\tau$ of the electron to the origin. See text for details. The classical curve is presented both with (dashed curve) and without (solid curve) inclusion of the magnetic-fieldinduced threshold shift, $\frac{1}{2} \hbar \omega_{B}$, corresponding to the quantummechanical lowest energy of the electron in the $B$ field. 


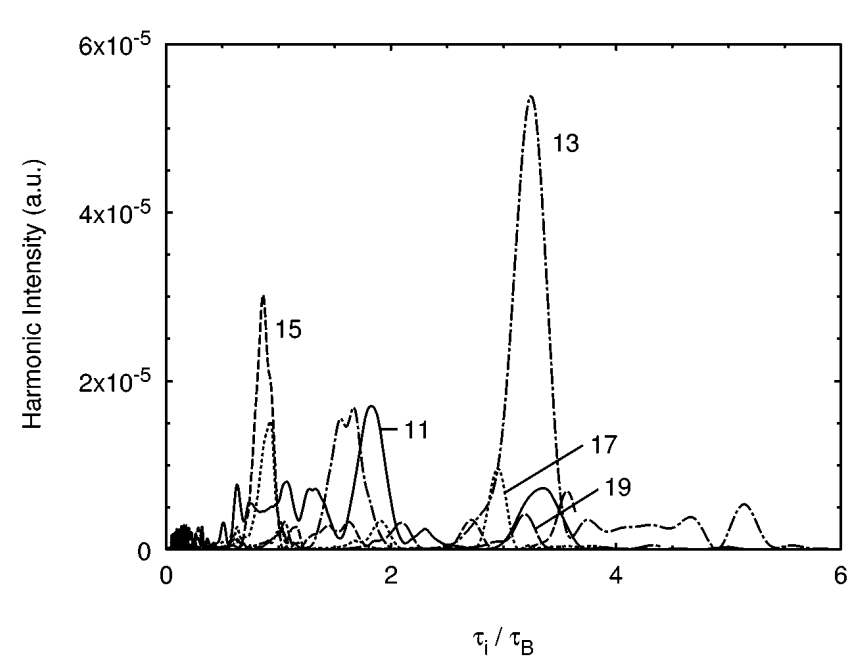

FIG. 4. Harmonic intensities for $11 \leq N \leq 19$ plotted in dimensionless units $\tau_{i}(N) / \tau_{B}$, where $i=1$ for $15 \leq N \leq 19$ and $i=2$ for $N=11,13$. $\tau_{1}\left(\tau_{2}\right)$ is the classically calculated first (second) shortest period for the return of the electron to the origin under the influence of the laser field, and $\tau_{B}$ is the cyclotron period. (Curve labeling is the same as in Fig. 2.)

by $\tau_{B}$. The dimensionless variables $\tau_{i}(N) / \tau_{B}, i=1,2$, measure the period $\tau_{i}(N)$ for the first or second return of the electron under the influence of the laser in units of $\tau_{B}$. Whenever $\tau_{1}(N)$ or $\tau_{2}(N)$ are close to an integer multiple of $\tau_{B}$, there is a revival of intensity in the emitted harmonics; moreover the interval between revivals is approximately $\tau_{B}$. Our interpretation of this result is that the intensity of harmonics peaks whenever the wave packet amplitudes for motion perpendicular to and along the $z$ axis coincide. (Exact coincidence is not expected, owing to the spatial width of quantum wave packets.) Indeed, our calculations for $\tau_{i}(N=13)$ for $1 \leq i \leq 6$ show that $\tau_{2} \approx 3 \tau_{B}, \tau_{4} \approx 5 \tau_{B}, \tau_{5} \approx 6 \tau_{B}$, and $\tau_{6} \approx 7 \tau_{B}$, implying that the high intensity of the 13th harmonic may stem from constructive interference of contributions associated with a large number of classical orbits along the $z$ axis with return times $\tau$ that are multiples of $\tau_{B}$.

Figures 3 and 4 demonstrate that, by using only a crude classical analysis, we can predict for which values of $B$ particular harmonics may be emitted with high efficiency. These are a second set of experimentally verifiable predictions of the "three-step model" [3,4] in addition to the well-known prediction for the harmonic cutoff, $N_{\max }$. Similar predictions for magnetic-fieldinduced revivals have been given for photodetachment of $\mathrm{H}^{-}$in parallel $\mathbf{E}$ and $\mathbf{B}$ fields using both short and long pulse lasers [15]. In that case, it is the static electric field $\mathbf{E}$ which reflects photodetached electron wave packets back toward the $\mathrm{H}$ atom and the focus is on the final, photodetached state. In the present case, these electron motions occur in the intermediate state and their influence is reflected by the intensities of the generated harmonics.

In conclusion, we have shown that it is possible to control high harmonic generation in a linearly polarized laser field by adding a parallel static magnetic field. For particular values of $B$, the intensity of particular harmonics can be increased by orders of magnitude. Using a $\mathrm{CO}_{2}$ laser and the $\mathrm{H}^{-}$ion in our examples, we have shown that the most important of these intensity maxima can be reached with magnetic field strengths comparable to those experimentally available at present. We have also explained, using the classical three-step model, that the positions of the most intense maxima correspond to values of $B$ for which an integer multiple of the classical period for motion perpendicular to the magnetic field is equal to the return time of the ionized electron to the atomic core. We interpret this fact to mean that the harmonic intensity has a maximum if the electronic wave packet is at the atomic core both in the parallel and in the perpendicular directions at the same time.

This work was supported in part by the National Science Foundation under Grant No. PHY-9722110.

*On leave from Faculty of Science and Mathematics, Department of Physics, University of Sarajevo, Zmaja od Bosne 35, 71000 Sarajevo, Bosnia and Herzegovina.

[1] Super-Intense Laser-Atom Physics IV, edited by H. G. Muller and M. V. Fedorov (Kluwer, Dordrecht, 1996).

[2] Multiphoton Processes - 1996, edited by P. Lambropoulos and H. Walther, IOP Conf. Proc. No. 154 (Institute of Physics and Physical Society, Bristol, 1997).

[3] K. C. Kulander, K. J. Schafer, and J.L. Krause, in Super-Intense Laser-Atom Physics, edited by B. Piraux, A. L'Huillier, and K. Rzążewski, NATO Advanced Study Institutes, Ser. B, Vol. 316 (Plenum, New York, 1993), p. 95.

[4] P. B. Corkum, Phys. Rev. Lett. 71, 1994 (1993).

[5] A. L'Huillier et al., Phys. Rev. A 48, R3433 (1993); M. Lewenstein et al., ibid. 49, 2117 (1994).

[6] M. Ivanov et al., Phys. Rev. Lett. 74, 2933 (1995); Ph. Antoine et al., Phys. Rev. A 54, R1761 (1996).

[7] S. Long, W. Becker, and J. K. McIver, Phys. Rev. A 52, 2262 (1995); D. B. Milošević and B. Piraux, ibid. 54, 1522 (1996); M. B. Gaarde, A. L'Huillier, and M. Lewenstein, ibid. 54, 4236 (1996).

[8] M. Q. Bao and A.F. Starace, Phys. Rev. A 53, R3723 (1996); A. Lohr, W. Becker, and M. Kleber, Laser Phys. 7, 615 (1997); B. Wang, X. Li, and P. Fu, J. Phys. B 31, 1961 (1998).

[9] T. Zuo et al., Phys. Rev. A 51, 3991 (1995); T. Zuo and A. D. Bandrauk, J. Nonlinear Opt. Phys. Mater. 4, 533 (1995); A. D. Bandrauk et al., Int. J. Quantum Chem. 64, 613 (1997).

[10] J.-P. Connerade and C.H. Keitel, Phys. Rev. A 53, 2748 (1996).

[11] Yu. B. Kudasov et al., JETP Lett. 68, 350 (1998).

[12] T. Ohmura and H. Ohmura, Phys. Rev. 118, 154 (1960).

[13] D. B. Milošević and A. F. Starace (to be published).

[14] C.-H. Park and A. F. Starace, Phys. Rev. A 29, 442 (1984).

[15] Q. Wang and A. F. Starace, Phys. Rev. A 51, 1260 (1995); 55, 815 (1997). 